\title{
ADMINISTRAÇÃO DE MEDICAMENTOS - CARGA DE TRABALHO DA EQUIPE DE ENFERMAGEM EM UNIDADE DE INTERNAÇÃO CLÍNICA
}

\author{
Angélica Kreling', Ana Maria Müller de Magalhães²
}

RESUMO: Objetivos: verificar a quantidade e tipo de medicamentos prescritos e administrados por técnicos de enfermagem em unidade de internação e discutir suas implicações na carga de trabalho da enfermagem e na segurança dos pacientes. Método: estudo transversal exploratório realizado em três unidades de internação clínica de um hospital universitário do sul do país, entre dezembro de 2014 e março de 2015. A amostra foi de 162 escalas de trabalho de pacientes atribuídos aos profissionais de enfermagem. Os dados foram coletados com instrumento específico e analisados por meio de estatística descritiva. Resultados: o número médio de doses prescritas por turno/técnico foi de $60,45(\mathrm{dp}=20,26)$ e administradas foi de $34,50(\mathrm{dp}=15,64)$. Identificouse 16 diferentes tipos de vias para administração dos fármacos. Conclusão: o número e tipo de medicamentos prescritos e administrados geram implicações na carga de trabalho da equipe e contribuem para risco de erros. DESCRITORES: Sistemas de medicação no hospital; Carga de trabalho; Segurança do paciente; Enfermagem.

\section{MEDICATION ADMINISTRATION - NURSING WORKLOAD IN CLINICAL INPATIENT UNITS}

ABSTRACT: Objectives: To verify the number and types of drugs prescribed and administered by nursing technicians in inpatient units and discuss its implications for nursing workload and patient safety. Method: exploratory cross-sectional study carried out in three inpatient units of a university hospital in southern Brazil from December 2014 to March 2015. The sample consisted of 162 work schedules of nurses that provided direct care to patients in the referred units. Data was collected with a tool designed for this purpose and analyzed with descriptive statistics. Results: the average number of doses prescribed per shift/technician was 60.45 $(s d=20.26)$ and administered was $34.50(s d=15.64)$. Sixteen different types of routes for administering the drugs were identified. Conclusion: The number and types of drugs prescribed and administered impact nursing workload and increase the risk of errors. DESCRIPTORS: Medication systems in the hospital; Workload; Patient safety; Nursing.

\section{ADMINISTRACIÓN DE MEDICAMENTOS - CARGA DE TRABAJO DEL EQUIPO DE ENFERMERÍA EN UNIDAD DE INTERNACIÓN CLÍNICA}

RESUMEN: Objetivos: verificar la cantidad y tipo de medicamentos prescriptos y administrados por auxiliares de enfermería en unidad de internación, y discutir sus implicaciones en la carga de trabajo de enfermería y en la seguridad de los pacientes. Método: Estudio transversal exploratorio, realizado en tres unidades de internación clínica de hospital universitario del sur de Brasil, entre diciembre de 2014 y marzo de 2015. Muestra de 162 escalas de trabajo de pacientes atribuidas a profesionales de enfermería Datos recolectados con instrumento específico, analizados por estadística descriptiva. Resultados: El número promedio de dosis prescriptas por turno/auxiliar fue de 60,41 ( $S D=20,26)$, y el de administradas, 34,50 ( $S D=15,64)$. Fueron identificadas 16 tipos de vías de administración para los fármacos. Conclusión: El número y tipo de medicamentos prescriptos y administrados genera implicancias en la carga de trabajo del equipo e incrementan el riesgo de errores.

DESCRIPTORES: Sistemas de Medicación en Hospital; Carga de Trabajo; Seguridad del Paciente; Enfermería.

${ }^{1}$ Enfermeira. Mestranda em Enfermagem. Universidade Federal do Rio Grande do Sul. Porto Alegre, RS, Brasil.

${ }^{2}$ Enfermeira. Doutora em Enfermagem. Docente de Enfermagem da Universidade Federal do Rio Grande do Sul. Porto Alegre, RS, Brasil. 


\section{INTRODUÇÃO}

As práticas de enfermagem, no cenário atual, vêm sendo constantemente associadas pela mídia a eventos relacionados à administração de medicamentos nos hospitais e em outros serviços de saúde. Embora este seja um processo multidisciplinar, a equipe de enfermagem possui grande responsabilidade no manejo da farmacoterapia e na garantia da segurança do paciente, pois desempenha papel central neste cuidado ${ }^{(1-4)}$.

A administração de medicamentos está intrinsecamente relacionada à carga de trabalho da equipe de enfermagem, visto que é tida como uma atividade preponderante no turno de trabalho ${ }^{(5)}$, ao mesmo tempo em que a sobrecarga é considerada um item colaborador para a ocorrência de erros na administração de medicamentos ${ }^{(1,6-7)}$. Além disso, a gestão e administração de medicamentos vêm se tornando uma atividade cada vez mais complexa na organização dos cuidados aos pacientes. $\mathrm{O}$ aumento da gravidade dos pacientes, a diminuição dos tempos de permanência e o aumento do número de medicações prescritas para os pacientes são fatores que têm sido considerados como contribuintes para o aumento da complexidade e das demandas de trabalho da equipe de enfermagem ${ }^{(3)}$.

Estima-se que $40 \%$ do tempo de enfermagem em unidades clínicas esteja relacionado ao processo de administração de medicamentos ${ }^{(7)}$. A alta demanda de envolvimento da equipe de enfermagem com o processo de medicação também foi destacada em recente estudo nacional ${ }^{(5)}$, o qual apontou que os profissionais reconhecem a administração de medicamentos como um dos principais fatores relacionados à alta carga de trabalho nas unidades e que pode acarretar riscos para a segurança dos pacientes. Os resultados demonstraram a relação da carga de trabalho com o aumento do número de pacientes por profissional, o que pode implicar no aumento do número de medicamentos a serem administrados por turno de trabalho a cada paciente.

Apesar de iniciativas para que se tenha administração segura de medicamentos no processo de trabalho da enfermagem, como a adoção dos itens de verificação a serem seguidos, denominados os "certos" da administração - paciente certo, medicamento certo, via, hora e dose certas, além do registro correto, indicação acertada, forma de apresentação e resposta corretas ${ }^{(8-9)}$, as falhas e os erros no processo de medicação estão entre os eventos adversos mais comuns nos hospitais ${ }^{(10-11)}$.

Essas falhas e os erros de medicação podem ocorrer decorrentes de falhas ativas, como o descuido ao fazer/seguir uma prescrição, e de condições latentes ${ }^{(12)}$, como a carga de trabalho e a falta de pessoal, cabendo à equipe de enfermagem a responsabilidade de garantir segurança ao paciente, posto que é a responsável em preparar e aplicar o fármaco no paciente, sendo a ponta final deste processo e detendo a última oportunidade de evitar e interceptar erros ${ }^{(13)}$.

O conceito de carga de trabalho adotado neste estudo baseia-se em várias pesquisas que têm buscado investigar a associação entre a carga de trabalho da equipe de enfermagem e os resultados de segurança do paciente, mediante o estudo do número de pacientes atribuídos a cada enfermeiro ou técnico de enfermagem em cada plantão, assim como das condições do ambiente de trabalho desses profissionais $^{(5,14-16)}$.

Diante dessas considerações, entende-se que essa pesquisa pode contribuir para o conhecimento da questão cada vez mais presente no cotidiano de trabalho dos profissionais de enfermagem: qual a quantidade e o tipo de medicamentos administrados por profissional de enfermagem em seu turno de trabalho?

Neste compasso, o estudo tem como objetivo verificar a quantidade e o tipo de medicamentos prescritos e administrados por funcionário em unidade de internação clínica e discutir suas implicações na carga de trabalho da equipe de enfermagem e na segurança de pacientes internados.

\section{MÉTODO}

Estudo transversal exploratório realizado em três unidades de internação clínica de um hospital universitário do sul do país. A população foi composta pelos profissionais de enfermagem atuantes nas unidades em estudo e os pacientes internados nas mesmas. Utilizou-se a técnica de amostragem 
aleatória sistemática, iniciando-se por um sorteio das escalas de trabalho em cada turno e alternando a sequência a cada nova coleta, seguindo-se os números ímpares ou pares da escala.

A amostra constituiu-se em 162 escalas de técnicos de enfermagem (TE), distribuídas nas três unidades, sendo 18 escalas de TE por turno, considerando-se nível de confiança de 95\%, margem de erro de 3 e média (desvio padrão) de 35 (10) medicamentos administrados por técnico de enfermagem em cada escala no turno de trabalho, além do acréscimo de $10 \%$ para possíveis perdas, determinados através de estudo piloto.

O critério de inclusão foi definido pela atuação dos técnicos de enfermagem na unidade em que faziam parte da escala de pacientes. Foram excluídas da amostra as escalas de técnicos de enfermagem cujos pacientes atribuídos não se encontravam na unidade, durante procedimentos cirúrgicos ou diagnósticos, impossibilitando a consulta local dos seus prontuários.

A coleta de dados foi realizada por meio de preenchimento de instrumento específico e consulta aos prontuários dos pacientes internados, de cada escala de técnico de enfermagem sorteada, no período de dezembro de 2014 a março de 2015, com dados de identificação do paciente (prontuário e leito), número e tipo de medicamentos prescritos, número e tipo de medicamentos administrados e número de pacientes na escala de cada profissional. Os dados de caracterização dos pacientes incluídos no estudo - média de permanência, sexo, faixa etária, especialidades médicas e diagnósticos médicos foram coletados por meio de consulta ao banco de dados institucional IG-BSC.

Os dados foram analisados por meio de estatística descritiva, sendo as variáveis contínuas descritas pela média e desvio padrão e as variáveis categóricas descritas pela frequência absoluta e relativa. Para a análise inferencial de diferenças das médias de medicamentos prescritos e administrados por turno, utilizou-se a Análise de Variâncias (ANOVA) seguida do teste de comparações múltiplas com ajuste de Bonferroni, com nível de significância estabelecido em $5 \%$. A análise foi realizada no programa Statistical Package for the Social Science (SPPS), versão 18.0.

O projeto respeitou todos os preceitos éticos e foi aprovado pelo Comitê de Ética da instituição, sob número 12-0332/2012 e, pelo Comitê de Ética em Pesquisa com Seres Humanos, n. 926.626/2014. Para a pesquisa em prontuários de pacientes, foi utilizado o Termo de compromisso para utilização de dados (TCUD).

\section{RESULTADOS}

Dos dados provenientes do IG-BSC, visando a caracterizar o perfil dos pacientes internados nas unidades pesquisadas no período em estudo (Tabela 1), identificou-se que ocorreram 1148 internações nas três unidades, sendo a média de permanência para este grupo de pacientes 8,58 dias. A amostra caracterizou-se por 581 (50,61\%) pacientes do sexo masculino e predominância de indivíduos com 60 anos ou mais $561(48,87 \%)$. Os dois principais grupos de diagnósticos médicos foram aqueles relacionados às doenças do aparelho circulatório 325 (22,20\%) e neoplasias 316 (21,58\%).

Tabela 1 - Caracterização dos pacientes internados nas unidades pesquisadas. Porto Alegre, RS, Brasil, 2015 (continua)

\begin{tabular}{lcc} 
Variável & $\mathbf{n}(\mathbf{N}=\mathbf{1 1 4 8})$ & $\mathbf{\%}$ \\
\hline Sexo & & \\
\hline Masculino & 581 & 50,61 \\
\hline Feminino & 567 & 49,39 \\
\hline Faixa etária & & \\
\hline 13 a 29 anos & 96 & 8,36 \\
\hline 30 a 59 anos & 491 & 42,77 \\
\hline 60 anos ou mais & 561 & 48,87 \\
\hline
\end{tabular}




\begin{tabular}{lcc}
\hline Especialidade médica & & \\
\hline Medicina Interna & 473 & 41,2 \\
\hline Neurologia & 104 & 9,06 \\
\hline Oncologia & 102 & 8,89 \\
\hline Outros & 469 & 40,85 \\
\hline Saídas por CIDt & & \\
\hline Capítulo IX - Doenças do aparelho circulatório & 325 & 22,2 \\
\hline Capítulo II - Neoplasias [tumores] & 316 & 21,58 \\
\hline Capítulo XI - Doenças do aparelho digestivo & 135 & 9,22 \\
\hline Capítulo X - Doenças do aparelho respiratório & 130 & 8,88 \\
\hline Capítulo I - Algumas doenças infecciosas e parasitárias & 109 & 7,45 \\
\hline Capítulo XVIII - Sintomas, sinais e achados anormais de exames clínicos e de & 90 & 6,15 \\
laboratório, não classificados em outra parte & & \\
\hline Outros & 359 & 24,52
\end{tabular}

tn total de saídas por CID maior que o n total de especialidade médica devido ao paciente possuir mais de um diagnóstico.

A partir da análise dos dados, oriundos de 162 escalas de técnicos de enfermagem, obteve-se o número e o tipo de medicamentos prescritos e administrados por técnico de enfermagem e por turno, conforme descrito na Tabela 2.

Tabela 2 - Número de medicamentos prescritos e administrados por turno, por técnico de enfermagem e por paciente em unidades de internação clínica. Porto Alegre, RS, Brasil, 2015

\begin{tabular}{|c|c|c|c|}
\hline Variável & Média & dp $\neq$ & p§ \\
\hline Medicamentos prescritos por técnicot† & & & $<0,001$ \\
\hline Manhã & 60,94 & 16,61 & \\
\hline Tarde & 44,48 & 13,27 & \\
\hline Noite & 75,96 & 17,06 & \\
\hline Geral & 60,45 & 20,26 & \\
\hline Medicamentos administrados por técnicot† & & & $<0,001$ \\
\hline Manhã & 35,43 & 11,17 & \\
\hline Tarde & 20,2 & 7,76 & \\
\hline Noite & 47,87 & 12,85 & \\
\hline Geral & 34,5 & 15,64 & \\
\hline Medicamentos prescritos por pacienteł‡ & & & $<0,001$ \\
\hline Manhã & 10,18 & 2,33 & \\
\hline Tarde & 7,44 & 1,91 & \\
\hline Noite & 10,18 & 2,06 & \\
\hline Geral & 9,26 & 2,46 & \\
\hline Medicamentos administrados por paciente $\neq \ddagger$ & & & $<0,001$ \\
\hline Manhã & 5,92 & 1,7 & \\
\hline Tarde & 3,37 & 1,13 & \\
\hline Noite & 6,41 & 1,58 & \\
\hline Geral & 5,23 & 2 & \\
\hline
\end{tabular}

‡Desvio padrão; §Nível descritivo amostral da ANOVA, seguida de comparações múltiplas com ajuste de Bonferroni; ††Diferença significativa entre os três turnos $(p<0,05)$; ‡łDiferença significativa do turno da tarde com os demais turnos $(\mathrm{p}<0,05)$. 
Observa-se que o turno da noite é aquele que concentra o maior número de medicamentos prescritos e administrados por técnico de enfermagem. Ressalta-se que este turno compreende 12 horas de trabalho, com maior intervalo de tempo para a administração de medicamentos. Além disso, verifica-se diferença significativa entre os turnos de trabalho, por meio da análise inferencial com o teste de Bonferroni.

Em relação ao tipo de medicamento prescrito e administrado, encontram-se 16 diferentes vias para administração dos fármacos. Foi constatada a maior média de medicamentos orais nos três turnos, seguido daqueles de via endovenosa, predominantemente prescritos para administração conforme "se necessário" (SN), em casos de analgesia, distúrbios gastrointestinais e de hipertermia, além da antibioticoterapia (Tabela 3).

Tabela 3 - Tipo de medicamentos prescritos e administrados por turno em unidades de internação clínica. Porto Alegre, RS, Brasil, 2015

\begin{tabular}{|c|c|c|c|c|c|c|}
\hline Tipo de via & Média & $d p$ & Média & $d p$ & Média & dp \\
\hline \multicolumn{7}{|l|}{ Via oral } \\
\hline Prescritos & 19,19 & 7,71 & 10,28 & 5,33 & 21,59 & 7,95 \\
\hline Prescritos SN & 6,11 & 2,95 & 5,85 & 2,45 & 7,46 & 3,62 \\
\hline Administrados & 17,44 & 6,7 & 9,7 & 5,2 & 20,8 & 7,35 \\
\hline \multicolumn{7}{|l|}{ Via endovenosa } \\
\hline Prescritos & 5,51 & 2,84 & 4,87 & 2,64 & 9,19 & 5,25 \\
\hline Prescritos SN & 12,37 & 4,08 & 12,76 & 4,58 & 15,11 & 3,99 \\
\hline Administrados & 6,06 & 3,1 & 5,32 & 2,67 & 10,58 & 5,76 \\
\hline \multicolumn{7}{|l|}{ Via subcutânea } \\
\hline Prescritos & 5,53 & 1,97 & 2,34 & 1,14 & 5,76 & 1,9 \\
\hline Prescritos SN & 2,04 & 1,11 & 2,09 & 1,24 & 2,4 & 1,43 \\
\hline Administrados & 5,7 & 2,07 & 2,32 & 1,28 & 5,85 & 1,98 \\
\hline \multicolumn{7}{|l|}{ Via intramuscular } \\
\hline Prescritos & 1 & --- & 1,14 & 0,38 & --- & --- \\
\hline Prescritos SN & 1,56 & 0,73 & 1,3 & 0,48 & 1,25 & 0,46 \\
\hline Administrados & --- & --- & 1 & --- & 1 & --- \\
\hline \multicolumn{7}{|l|}{ Via sonda } \\
\hline Prescritos & 5,06 & 3,76 & 3,17 & 2,25 & 6,49 & 3,47 \\
\hline Prescritos SN & 1,5 & 0,69 & 1,3 & 0,57 & 2,12 & 1,77 \\
\hline Administrados & 4,79 & 3,46 & 3,15 & 2,18 & 6,6 & 3,42 \\
\hline \multicolumn{7}{|l|}{ Via tópica } \\
\hline Prescritos & 2,09 & 1,52 & 1,54 & 0,95 & 2,1 & 1,47 \\
\hline Prescritos SN & 1 & --- & 1 & --- & 1 & --- \\
\hline Administrados & 1,85 & 1,18 & 1,61 & 0,84 & 1,79 & 1,21 \\
\hline \multicolumn{7}{|l|}{ Via inalatória } \\
\hline Prescritos & 3,23 & 2,44 & 1,55 & 0,77 & 4,49 & 3,73 \\
\hline Prescritos SN & 1,38 & 0,52 & 1,2 & 0,45 & 1,75 & 0,96 \\
\hline Administrados & 3,25 & 2,3 & 1,52 & 0,74 & 4,31 & 3,68 \\
\hline \multicolumn{7}{|l|}{ Soro/Soluções } \\
\hline Prescritos & 1,9 & 0,97 & 1,9 & 1,08 & 2,19 & 1,31 \\
\hline Prescritos SN & 1,64 & 1,33 & 1,81 & 1,15 & 1,88 & 1,17 \\
\hline Administrados & 1,43 & 0,7 & 1,28 & 0,54 & 1,97 & 0,1 \\
\hline \multicolumn{7}{|l|}{ Outros§§ } \\
\hline Prescritos & 1,64 & 0,81 & 2 & 0,63 & 3,73 & 2,49 \\
\hline Prescritos SN & 1,28 & 0,46 & 1,48 & 0,67 & 1,39 & 0,5 \\
\hline Administrados & 1,67 & 0,71 & 1,33 & 0,52 & 3,33 & 2,47 \\
\hline
\end{tabular}

$\S \S \mathrm{VC}=$ via ocular, $\mathrm{VR}=$ via retal, $\mathrm{SL}=$ sublingual, $\mathrm{GU}=$ geniturinário, $\mathrm{VA}=$ via auricular, $\mathrm{VN}=$ via nasal, $\mathrm{ID}=$ via intradérmica e $\mathrm{PD}=$ peridural. 
Os medicamentos de uso por via inalatória, via tópica e via sonda apresentam os maiores percentuais de administração em relação aos prescritos (Tabela 4), considerando que nestas vias verifica-se que são poucos os medicamentos prescritos caso necessário.

Tabela 4 - Percentual médio dos medicamentos administrados em relação aos prescritos. Porto Alegre, RS, Brasil, 2015

\begin{tabular}{lcccccc} 
& \multicolumn{2}{c}{ Manhã } & \multicolumn{2}{c}{ Tarde } & Noite \\
\hline Tipo de via & Média & dp & Média & dp & Média & dp \\
\hline Via oral & 67,97 & 13,62 & 57,99 & 15,78 & 73,04 & 13,42 \\
\hline Via endovenosa & 32,58 & 11,72 & 30,22 & 12,81 & 41,82 & 15,4 \\
\hline Via subcutânea & 79,29 & 14,98 & 58,39 & 22,6 & 75,2 & 15,71 \\
\hline Via intramuscular & --- & --- & 76,19 & 30,21 & 100 & --- \\
\hline Via sonda & 79,97 & 18,27 & 82,05 & 17,68 & 86,27 & 13,21 \\
\hline Via tópica & 89,8 & 18,18 & 94,78 & 14,73 & 87 & 22 \\
\hline Via inalatória & 90,79 & 16,09 & 91,95 & 18,16 & 93,34 & 13,53 \\
\hline Soro/Soluções & 52,42 & 29,13 & 43,96 & 19,33 & 57,68 & 22,66 \\
\hline Outrost+† & 78,89 & 25,22 & 72,22 & 31,03 & 74,22 & 22,76
\end{tabular}

†+†VC = via ocular, $\mathrm{VR}=$ via retal, $\mathrm{SL}=$ sublingual, $\mathrm{GU}=$ geniturinário, $\mathrm{VA}=$ via auricular, $\mathrm{VN}=$ via nasal, $\mathrm{ID}=$ via intradérmica e $\mathrm{PD}=$ peridural.

A partir das escalas de trabalho, verificou-se o número médio de pacientes por técnico/turno, com uma média de 5,98 (dp = 0,90) pacientes/técnico no turno da manhã, 5,98 (dp = 0,83) no turno da tarde e $7,44(d p=0,60)$ no turno da noite.

\section{- DISCUSSÃO}

Ao analisar o perfil dos pacientes internados, apurou-se que houve predominância de indivíduos idosos, com 60 anos ou mais, e que os principais diagnósticos médicos estavam relacionados a doenças do aparelho circulatório e a neoplasias. Os achados de uma pesquisa brasileira, que caracterizou o perfil das internações hospitalares de idosos, indicam como principais causas de internação as doenças de ordem circulatória e respiratória, bem como as neoplasias ${ }^{(17)}$.

No âmbito internacional, um estudo americano que teve como objetivo determinar os fatores preditivos de erros de medicação e descrever o tipo e número destes erros experienciados por idosos hospitalizados, encontrou resultados semelhantes com a presente investigação. Dentre os motivos de hospitalização neste grupo de pacientes, estavam os distúrbios do sistema circulatório 2903 (28,5\%) e as neoplasias 1406 (13,8\%). Além disso, como fatores preditivos de falhas neste processo de medicação, os autores apontaram o tipo de fármacos e o número total de medicamentos que os pacientes recebem durante a internação (polifarmácia) ${ }^{(18)}$.

Verificou-se que o número médio de medicamentos prescritos nas escalas de trabalho dos técnicos foi de $60,45(\mathrm{dp}=20,26)$, sendo 9,26 $(\mathrm{dp}=2,46)$ o número médio de doses prescritas por paciente. Estudo observacional mostrou resultado semelhante, com média de 9,7 medicamentos prescritos por paciente e variação de 0 a 20 fármacos ${ }^{(19)}$. Entretanto, os achados de outras pesquisas divergem dos números encontrados, demonstrando uma ampla variação de número de doses prescritas por paciente em diferentes contextos ${ }^{(11,20)}$.

Essas variações em achados de estudos locais e internacionais quanto ao número de medicamentos prescritos por paciente, apontam a necessidade de maiores estudos para descrever a magnitude desse fenômeno no cenário brasileiro e reforçam a importância da presente investigação. 
A alta carga de trabalho, considerando-se o número de medicamentos que necessitam ser lidos e interpretados para serem preparados e administrados, além das diferentes atividades no turno de trabalho, pode interferir na dinâmica e no processo seguro de medicamentos, contribuindo para a ocorrência de erros.

Em relação aos medicamentos administrados por técnico em seu turno de trabalho, verificou-se que a média foi de 34,50 ( $\mathrm{dp}=15,64)$, sendo 5,23 (dp = 2,00) o número médio de doses administradas por paciente. Considera-se que esses achados indicam um elevado número de medicamentos que cada profissional deve preparar e administrar em seu turno de trabalho, devendo ser um aspecto de preocupação e maior investigação para a segurança dos processos assistenciais. Em estudo sobre eventos adversos relacionados a medicamentos, no qual se adotou a técnica de observação "naïve", encontrou-se que $27,30 \%$ dos pacientes observados receberam uma dose de medicamento, $17,20 \%$ duas doses, $11,60 \%$ três doses, $10,60 \%$ quatro doses e $33,30 \%$ cinco doses ou mais com um máximo de 31 doses $^{(21)}$.

Entende-se que o número de doses administradas de medicamentos e o número de pacientes sob os cuidados dos profissionais de enfermagem são componentes importantes para avaliar a demanda de trabalho da equipe, frente às necessidades dos pacientes. Os erros de medicação foram significativamente associados ao aumento do número de pacientes atribuídos às enfermeiras ${ }^{(20,22)}$ e foi evidenciado que a maior frequência desses erros nos hospitais ocorrem em unidades de internação ${ }^{(23)}$. As características dos usuários dessas unidades, como pacientes fisicamente vulneráveis com grande quantidade de medicações e alta demanda de trabalho exigida da equipe de enfermagem, foram destacadas como possíveis causas dessas falhas ${ }^{(23)}$.

Quanto ao tipo de via, observou-se que os medicamentos orais, seguido dos endovenosos e dos administrados via sonda são os tipos mais prescritos. Estudos observacionais demonstraram semelhantes resultados e relataram que as principais vias empregadas são as vias parenteral, oral e enteral ${ }^{(19-20)}$.

Dentre os fatores causais de erros, encontram-se o aumento do número de medicamentos administrados, tanto daqueles que são prescritos como dos que são prescritos se necessário, e a diversidade de dosagens e formas de apresentação dos fármacos, principalmente quando estes são administrados conjuntamente ${ }^{(11,19)}$. Cabe destacar que, de acordo com a Política Nacional de Segurança do Paciente, a prescrição de medicamentos "se necessário" deve ser acompanhada de informações acerca de sua dose, posologia e condições de uso com o objetivo de prevenir possíveis interações e incompatibilidades farmacológicas ${ }^{(8)}$.

Pesquisas atuais evidenciam que os erros ocorrem na proporção de um para cada quatro medicamentos administrados ${ }^{(19)}$ e que há um aumento de 3,32 vezes no risco de ocorrência quando são prescritos três ou mais medicamentos por dia, além de estabelecer relação entre o tempo de permanência do paciente internado (quatro dias ou mais) e a ocorrência de erros ${ }^{(11)}$.

Considerando-se os dados da literatura e os achados do presente estudo, pondera-se que o conhecimento acerca do número de medicamentos prescritos e administrados por paciente, assim como o número de pacientes em cada escala de trabalho dos profissionais de enfermagem, são variáveis importantes para avaliar a carga de trabalho da equipe e pensar em estratégias para adequar a organização desses processos de cuidado. A identificação dos horários de maior incidência, assim como o tipo e vias de administração de medicamentos, pode contribuir para planejar a distribuição da equipe de enfermagem e diminuir os riscos de erros de medicação.

Como verificado nos dados da pesquisa, foi significativa a diferença no número de medicações administradas nos diferentes turnos, sinalizando a necessidade de avaliar a adequação de pessoal para atender essa demanda de cuidado dos pacientes. O maior número de medicamentos e o maior número de pacientes por profissional de enfermagem identificado no turno noturno merece um olhar mais atento para o planejamento de pessoal e organização dos processos assistenciais.

Como limitações do estudo, podemos destacar que esta pesquisa foi realizada somente em unidades de internação clínica e em uma única instituição, não permitindo a generalização dos achados. Sabe-se que em outros cenários, como as unidades de internação cirúrgica e as unidades de terapia intensiva, as demandas de trabalho decorrentes dos cuidados com a farmacoterapia do paciente podem ser 
diferentes e, possivelmente, possam apresentar outros resultados.

\section{- CONCLUSÃO}

Os achados deste estudo demonstraram um elevado número e diferentes tipos de medicamentos que devem ser preparados e administrados pelo profissional de enfermagem a cada turno de trabalho $34,50(\mathrm{dp}=15,64)$, sendo que a maior concentração se deu nos turnos da noite e manhã, respectivamente.

O número, horário, tipo e vias de administração de medicamentos, juntamente com o número de pacientes sob os cuidados dos profissionais de enfermagem nos diferentes turnos de trabalho, são fatores importantes a serem considerados na carga de trabalho da equipe de enfermagem e podem ter implicações na segurança dos pacientes por representar maior risco na ocorrência de erros.

\section{- REFERÊNCIAS}

1. Praxedes MFS, Telles Filho PCP. Erros e ações praticadas pela instituição hospitalar no preparo e administração de medicamentos. Reme, Rev. Min. Enferm. [Internet] 2011;15(3) [acesso em 18 fev 2017]. Disponível: http://www. reme.org.br/artigo/detalhes/52.

2. Franco JN, Ribeiro G, D'Innocenzo M, Barros BPA. Percepção da equipe de enfermagem sobre fatores causais de erros na administração de medicamentos. Rev. bras. enferm. [Internet] 2010;63(6) [acesso em 18 fev 2017]. Disponível: http://dx.doi.org/10.1590/S0034-71672010000600009.

3. Bourbonnais FF, Caswell W. Teaching successful medication administration today: more than just knowing your 'rights'. Nurse Educ Pract. [Internet] 2014;14(4) [acesso em 18 fev 2017]. Disponível: http://dx.doi.org/10.1016/j. nepr.2014.03.003.

4. Adhikari R, Tocher J, Smith P, Corcoran J, Mac Arthur J. A multi-disciplinary approach to medication safety and the implication for nursing education and practice. Nurse Educ Today. [Internet] 2014;34(2) [acesso em 18 fev 2017]. Disponível: http://dx.doi.org/10.1016/j.nedt.2013.10.008.

5. de Magalhães AMM, Dall'Agnol CM, Marck PB. Carga de trabalho da equipe de enfermagem e segurança do paciente - estudo com método misto na abordagem ecológica restaurativa. Rev. Latino-Am. Enfermagem. [Internet] 2013;21(n.esp) [acesso em 18 fev 2017]. Disponível: http://dx.doi.org/10.1590/S0104-11692013000700019.

6. Volpe CRG, Pinho DLM, Stival MM, Karnikowski MGO. Medication errors in a public hospital in Brazil. Br J Nurs. [Internet] 2014;23(11) [acesso em 18 fev 2017]. Disponível: http://dx.doi.org/10.12968/bjon.2014.23.11.552.

7. Armitage G, Knapman H. Adverse events in drug administration: a literature review. J Nurs Manag. [Internet] 2003;11(2) [acesso em 18 fev 2017]. Disponível: http://dx.doi.org/10.1046/j.1365-2834.2003.00359.x.

8. Ministério da Saúde (BR). Anexo 03: protocolo de segurança na prescrição, uso e administração de medicamentos. [Internet] 2013 [acesso em 18 fev 2017]. Disponível: http://www20.anvisa.gov.br/segurancadopaciente/index.php/ publicacoes/item/seguranca-na-prescricao-uso-e-administracao-de-medicamentos.

9. Elliott M, Liu Y. The nine rights of medications administration: an overview. Br J Nurs. [Internet] 2010;19(5) [acesso em 18 fev 2017]. Disponível: http://dx.doi.org/10.12968/bjon.2010.19.5.47064.

10. D'Amour D, Dubois CA, Tchouaket E, Clarke S, Blais R. The occurrence of adverse events potentially attributable to nursing care in medical units: cross sectional record review. Int J Nurs Stud. [Internet] 2014;51(6) [acesso em 18 fev 2017]. Disponível: http://dx.doi.org/10.1016/j.ijnurstu.2013.10.017.

11. Paranaguá TTB, Bezerra ALQ, dos Santos ALM, Silva AEBC. Prevalência e fatores associados aos incidentes relacionados à medicação em pacientes cirúrgicos. Rev. esc. enferm. USP. [Internet] 2014;48(1) [acesso em 18 fev 2017]. Disponível: http://dx.doi.org/10.1590/S0080-623420140000100005.

12. Reason J. Human error: models and management. BMJ. [Internet] 2000;(320) [acesso em 18 fev 2017 ]. Disponível: https://doi.org/10.1136/bmj.320.7237.768. 
13. Ferreira RA, Pangaio AMWS, Bernardes RR, de Lima SS. Segurança do paciente e os eventos adversos: erro profissional ou do sistema? Rede Cuid. Saúde. [Internet] 2014;8(3) [acesso em 18 fev 2017]. Disponível: http:// publicacoes.unigranrio.br/index.php/rcs/article/view/2033.

14. Aiken LH, Sermeus W, Van den Heede K, Sloane DM, Busse R, McKee M, et al. Patient safety, satisfaction, and quality of hospital care: cross sectional surveys of nurses and patient in 12 countries in Europe and the United States. BMJ. [Internet] 2012;(344) [acesso em 18 fev 2017]. Disponível: https://doi.org/10.1136/bmj.e1717.

15. Dubois CA, D'Amour D, Tchouaket E, Clarke S, Rivard M, Blais R. Associations of patient safety outcomes with models of nursing care organization at unit level in hospitals. Int J Qual Health Care. [Internet] 2013;25(2) [acesso em 18 fev 2017]. Disponível: http://dx.doi.org/10.1093/intqhc/mzt019.

16. Nogueira LS, Koike KM, Sardinha DS, Padilha KG, de Sousa RMC. Nursing workload in public and private intensive care units. Rev. bras. ter. intensiva. [Internet] 2013;25(3) [acesso em 18 fev 2017]. Disponível: http:// dx.doi.org/10.5935/0103-507X.20130039.

17. de Castro VC, Borghi AC, Mariano PP, Fernandes CAM, Mathias TAF, Carreira L. Perfil de internações hospitalares de idosos no âmbito do sistema único de saúde. Rev. Rene. [Internet] 2013;14(4) [acesso em 28 set 2017]. Disponível: http://www.revistarene.ufc.br/revista/index.php/revista/article/viewFile/1269/pdf.

18. Picone DM, Titler MG, Dochterman J, Shever L, Kim T, Abramowitz P, et al. Predictors of medication errors among elderly hospitalized patients. Am J Med Qual. [Internet] 2008;23(2) [acesso em 27 set 2017]. Disponível: http://dx.doi.org/10.1177/1062860607313143.

19. Härkänen $M$, Ahonen J, Kervinen $M$, Turunen $H$, Vehviläinen-Julkunen K. The factors associated with medication errors in adult medical and surgical inpatients: a direct observation approach with medication record reviews. Scand J Caring Sci. [Internet] 2015;29(2) [acesso em 23 fev 2017]. Disponível: http://dx.doi.org/10.1111/ scs.12163.

20. Jennings BM, Sandelowski M, Mark B. The nurse's medication day. Qual Health Res. [Internet] 2011;21(10) [acesso em 23 fev 2017]. Disponível: http://dx.doi.org/10.1177/1049732311411927.

21. Donaldson N, Aydin C, Fridman M, Foley M. Improving medication administration safety: using naïve observation to assess practice and guide improvements in process and outcomes. J Healthc Qual. [Internet] 2014;36(6) [acesso em 23 fev 2017]. Disponível: http://dx.doi.org/10.1111/jhq.12090.

22. Berdot S, Sabatier B, Gillaizeau F, Caruba T, Prognon P, Durieux P. Evaluation of drug administration errors in a teaching hospital. BMC Health Serv Res. [Internet] 2012;12(60) [acesso em 27 set 2017]. Disponível: https://doi. org/10.1186/1472-6963-12-60.

23. Forte ECN, Machado FL, de Pires DEP. A relação da enfermagem com os erros de medicação: uma revisão integrativa. Cogitare Enferm. [Internet] 2016;21(5) [acesso em 27 set 2017]. Disponível: http://dx.doi.org/10.5380/ ce.v21i5.43324. 